\title{
Chronic Pancreatitis: Laser Technologies for Double Balloon Enteroscopy of Pancreatojejunostomy Complications and Reducing the Peripheral Ductal Hypertension
}

\author{
Aliaksandr Varabei ${ }^{1,}$, Anatoli Shuleika ${ }^{1}$, Yury Vizhinis ${ }^{1}$, Yury Arlouski ${ }^{1}$, Natalia Lagodich ${ }^{2}$ \\ ${ }^{1}$ Belarusian Medical Academy of Postgraduate Education, Minsk, Belarus \\ ${ }^{2}$ Minsk Regional Clinical Hospital, Endoscopy Unit, Minsk, Belarus
}

Email address:

dept-surg@hotmail.com (A. Varabei)

\section{To cite this article:}

Aliaksandr Varabei, Anatoli Shuleika, Yury Vizhinis, Yury Arlouski, Natalia Lagodich. Chronic Pancreatitis: Laser Technologies for Double Balloon Enteroscopy of Pancreatojejunostomy Complications and Reducing the Peripheral Ductal Hypertension. Journal of Surgery.

Vol. 3, No. 4, 2015, pp. 32-38. doi: 10.11648/j.js.20150304.11

\begin{abstract}
Introduction: Drainage procedures are conventional modifications of surgical treatment for chronic pancreatitis (CP). Nevertheless, the relapse of chronic abdominal pain after the surgery occurs in more than $50 \%$ of cases. Aim: To explain the pathogenesis of intractable abdominal pain following the traditional surgery of CP and to propose new laser and double balloon enteroscopy (DBE) technologies for its relief. Materials and methods: Pancreatojejunoanastomosis (PJA) lumen was examined using the videoenteroscope EN-450P5/20 (Fujifilm group, Fujinon Co, Tokio, Japan) for the first time in the world. All 20 patients had had CP and underwent an elective surgery earlier. During 2010-2014, 198 resections were performed, with 159 $(80,3 \%)$ being laser drainage procedures. Results: DBE revealed strictures of PJA and open common bile duct, residual pancreatoliths in PJA lumen, and PJA leakage. After DBE laser lithotripsy of pancreatoliths in tributary ducts, a free outflow of pancreatic juice from the lumen was obtained. DBE findings were supported by the morphological study of excised pancreatic specimen, CT-scans before the surgery and the staining of PJA lumen. All these data suggest that $17,7 \%$ of CP patients along with magistral ductal hypertension had peripheral ductal hypertension (PDH), which seems to be a new cause of severe abdominalgia. The latter arises when pancreatoliths block the orifices of tributary ducts. For reducing the PDH, we have developed a new surgical procedure named «laser cylindric wirsungectomy» $(\mathrm{n}=26)$ and proposed our own classification of pancreatic duct stones. Conclusions: DBE has great opportunities for the diagnosis of complications and their mini-invasive treatment after pancreatojejunostomy (PJ). Laser technologies are considered to be a new approach to the effective open and mini-invasive surgical treatment of $\mathrm{CP}$.
\end{abstract}

Keywords: Double Balloon Enteroscopy, Pancreatojejunostomy, Laser, Lithotripsy

\section{Introduction}

The main causes of intractable pain in chronic pancreatitis (CP) are ductal hypertension and perineural inflammation in the pancreatic head $[1,2,3,4]$. The large duct form of $\mathrm{CP}$ is defined when the Wirsung ductal diameter is greater than 7 $\mathrm{mm}$ [5]. A small duct $\mathrm{CP}$ is defined as the main ductal diameter of $<3 \mathrm{~mm}$. J.Izbicki pointed out that in these cases «perineural leukocyte infiltration has been presumed to play a causative role in pain generation» [6]. Other authors assess an increased parenchymatous pressure as a reason of severe pain $[7,8]$. Experimental glandular pancreatic compartment syndrome is suspected to cause the pain in CP as well [9].

Some authors have proposed a conservative treatment for complicated $\mathrm{CP}$ including endoscopic pancreatic drainage and extracorporeal shock wave lithotripsy. Endoscopic lithotripsy of pancreatoliths may be a promising future technique [10,11]. To understand the cause of chronic abdominalgia and to develop an adequate treatment strategy for $\mathrm{CP}$, some clinical classifications were proposed during the last fifteen years $[12,13,14]$.

Routine drainage procedures (Pargtington-Rochelle, classic Frey) are not effective because of high recurrence of chronic intractable abdominal pain $[15,16]$. Nowadays, 
resection-drainage procedures (Beger, Berne's modification of Beger technique, and modified Frey) are more effective for reducing the pancreatic and biliary hypertension and relieving the pain syndrome caused by inflammatory masses located in the head of the pancreas $[17,18,19]$. J.Izbicki proposed the so-called «V-shaped longitudinal excision of the ventral pancreas» for the small duct disease [6]. Laser technologies, widely spread in other fields of surgery, can be used for the complicated CP surgery [20].

Double balloon enteroscopy (DBE) presents new opportunities for the visual assessment of the small intestine with its altered anatomy after various reconstructive operations. Mostly, they concern procedures on biliary ducts and the stomach: hepaticojejunostomy, gastrectomy, and Whipple procedure $[21,22]$. Moreover, there have been single reports concerning mini-invasive DBE procedures: balloon dilatation, stenting, argon-plasma and laser vaporization of strictures, biopsy $[22,23,24]$. Unfortunately, the available literature has given no evidence of using DBE to diagnose the pathology of pancreatojejunoanastomosis (PJA) lumen so far and reports on performing mini-invasive procedures in this zone.

In this study, we assessed the efficacy of laser resection-drainage procedures for $\mathrm{CP}$ and the role of DBE in the diagnosis and treatment of the complications following the primary or repeated CP surgery. On the basis of the obtained data, we contribute to the ductal hypertension theory of chronic abdominal pain in CP. We have developed a new surgical approach for its relief and proposed a new classification of the pancreatic duct stones.

\section{Materials and Methods}

\subsection{Patients' Characteristics}

The types of currently used resection-drainage procedures for $\mathrm{CP}$ are given in Table 1.

For the primary surgery of CP, since 2012, we have developed, patented and carried out $(n=26)$ a laser longitudinal pancreatowirsungotomy and laser cylindric excision of the main pancreatic duct in the body of the pancreas named «cylindric wirsungectomy» $[25,26]$. The next stage was the local resection of the pancreatic head (Frey procedure) or its subtotal resection (Berne's modification of Beger procedure). First 77 patients were operated on randomly. In group $1 \quad(n=39)$, we resected the parenchyma of the pancreatic head and body by mono- and bipolar coagulation with additional ligation of pancreatoduodenal arteries branches. In group $2 \quad(\mathrm{n}=38)$, we used a high-energy three-wave laser «MUL» (Tetraedr, Belarus). This laser system allows working on three various wave lengths (1.06, 1.32 and 1.44 nanometers) with quick switching from wave to wave. For the resection of the pancreas, a laser beam with the wavelength of 1.32 nanometers and the power of 36-45 Watts was used. Since 2013, we have performed only laser resections of the pancreas $(n=121)$. In cases of biliary hypertension, the internal cholangiopancreatostomy (ChPS) was carried out $(\mathrm{n}=52)$.

The study was approved by local Ethics Committee. All patients signed the informed consent.

Table 1. Our experience in surgical treatment of ChP (2010-2014).

\begin{tabular}{lll}
\hline Procedure (N=198) & N total & N including \\
\hline Berne's modification of Beger technique: & 161 & 52 \\
opened common bile duct (ChPS) & & 9 \\
V-shaped resection of body (Izbicki) & & 26 \\
laser cylindricvirsungectomy* & 37 & \\
Other procedures & 20 & 6 \\
$\begin{array}{l}\text { DBE examination of PJA lumen: } \\
\text { miniinvasive laser procedures }\end{array}$ & & \\
\hline
\end{tabular}

*our patented proposal (2015).

\subsection{DBE Visualization of PJA Lumen and Mini-Invasive Procedures}

Since October 2010, in our Centre, we performed 20 transoral (antegrade) DBE examinations of PJA lumen through a Roux loop. The patients' age varied from 32 to 65 years. These patients underwent drainage $(n=6)$ and resection-drainage procedures $(n=12)$ for $C P$ and Whipple procedure $(\mathrm{n}=2)$ for pseudotumorous pancreatitis. The timing for DBE after the surgery varied from 3 to 24 months. The average duration of the DBE examination of the Roux loop and PJA lumen was $85 \pm 14 \mathrm{~min}$. The lumen of PJA was examined in $17(85,0 \%)$ of 20 patients.

\section{Results}

The laser excision of the pancreatic head and body during resection-drainage procedures allowed providing a stable, reliable hemostasis and reducing the intraoperative blood loss $(89 \pm 28 \mathrm{ml})$ as compared with pancreatic parenchyma resection by electrocoagulation $(311 \pm 41 \mathrm{ml})$. The additional pancreatic vessels ligation or sewing the tissue of the pancreas was not required after the laser resection.

The level of postoperative complications was significantly higher after electro surgical resections of the pancreas $(28,2 \%)$ versus the laser technology $(5,7 \%)[p<0,05]$. It is worth noting that there were no registered cases of postoperative bleeding and PJA leakage after laser resections.

During DBE $(\mathrm{n}=20)$, some delayed complications were revealed: PJA strictures after the Partington-Rochelle procedure (2); ChPS stricture (1) and PJA leakage (1) after the Berne's procedure; residual concrements in PJA lumen (3). The following mini-invasive procedures were performed: pancreatic parenchyma biopsy; laser lithotripsy of residual pancreatoliths in PJA lumen; laser vaporisation of ChPS stricture.

Peripheral ductal hypertension: a new cause of chronic abdominal pain. Four evidences. The terms «calcifying» pancreatitis, «calcification» or «calcinosis» of the pancreas that imply the calcification of the pancreatic tissue caused by necrotizing pancreatitis are still disputable. Our experience in laser resection-drainage procedures for $\mathrm{CP}$ allows assuming 
that the foci of calcification in the pancreas are practically always concrements in the peripheral ductal system. Due to

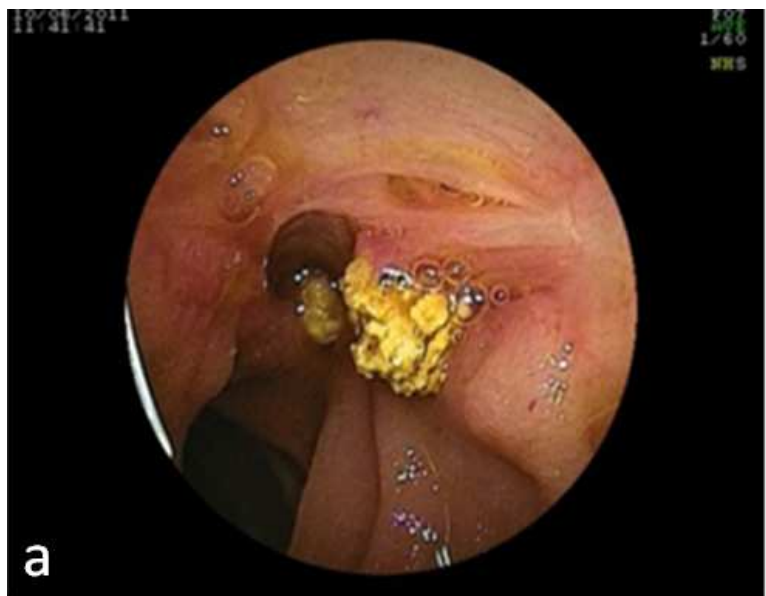

their corral-like shape (Figure 1), they are fixed in tributary ducts orifices and cause PDH.

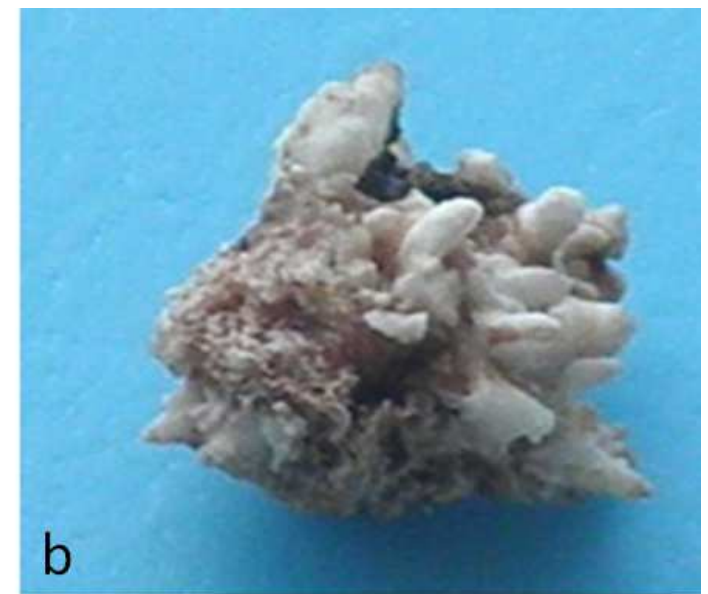

Figure 1. Sharp edges of the concrements: $a$-at the DBE examination of the PJA lumen (arrow); $b$-frommacrospecimen after a surgical treatment ofChP.

During the laser resection of the pancreatic parenchyma across the concrements, there was observed an effect of their lithotripsy accompanied by a typical sound. This made it possible to visualize even small concrements. After their extraction or lithotripsy, a free outflow of pancreatic juice through tributary ducts (of secondary or tertiary order) was always identified (Evidence 1 of PDH). These data are
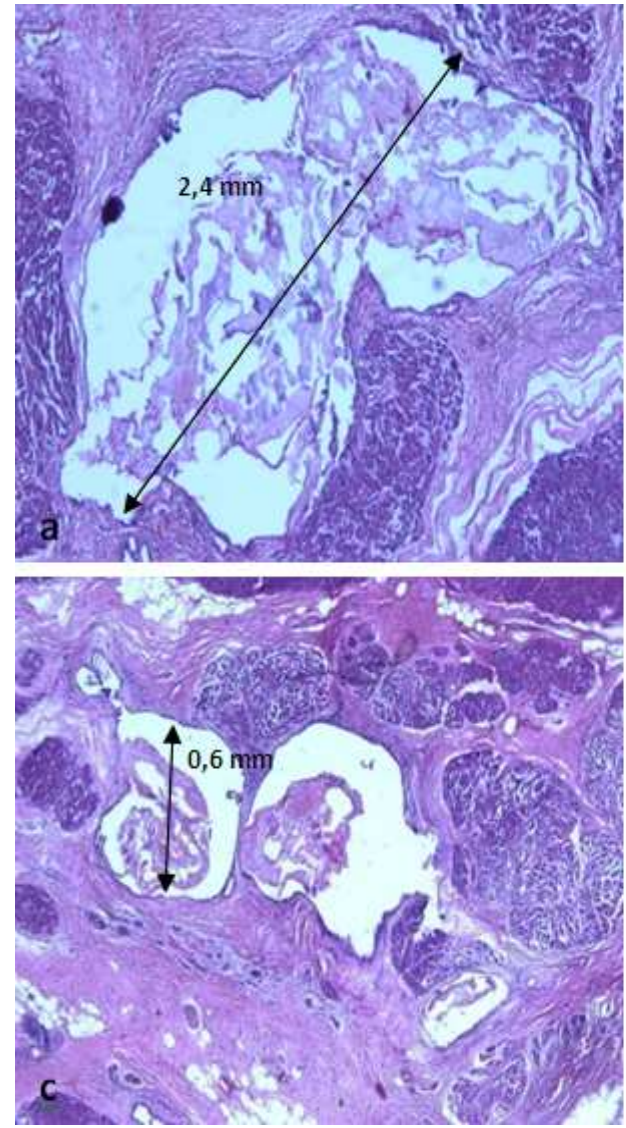

supported by the morphological study (Figure 2) of the pancreatic specimen (Evidence 2). Before the surgery, the dilation of the tributary ducts (Figure 3) due to strictures or concrements in their distal parts was registered on the CT-scans (Evidence 3). After the cylindric wirsungectomy (Figure 4), this dilation and PDH was reduced (Evidence 4).
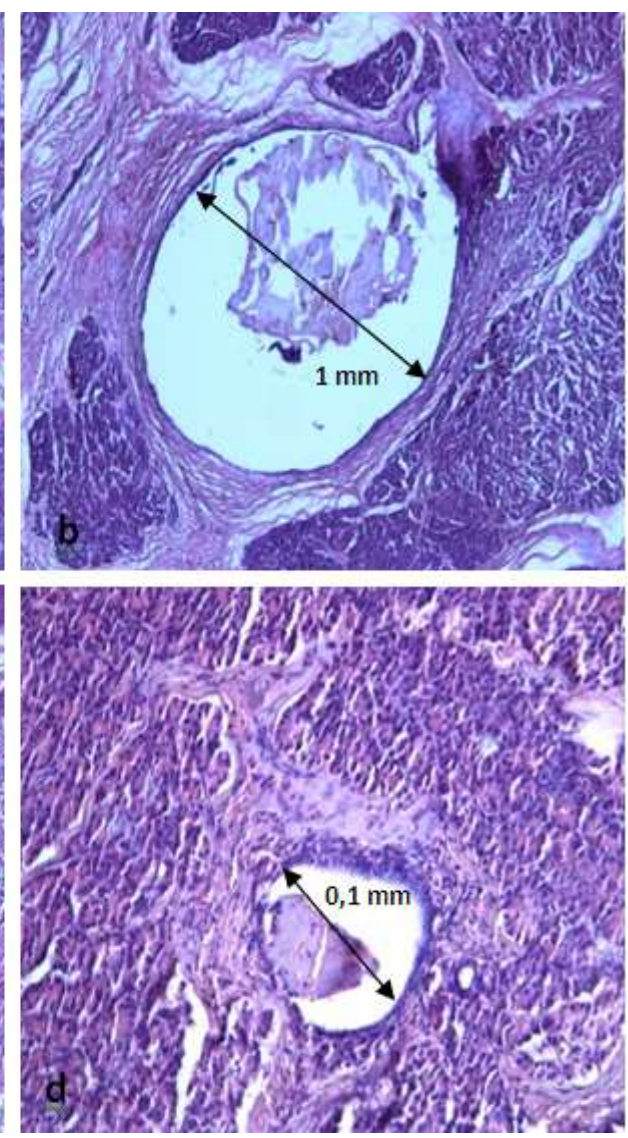

Figure 2. Own microphotos of the excised tissues of the pancreas head (hematoxylin and eosin staining after decalcification of surgical specimen): a,b,c,dpancreatoliths in the tributary ducts of various diameters (enhancement: $a, b, c-x 1 / 50 ; d-x 1 / 200$ ). 


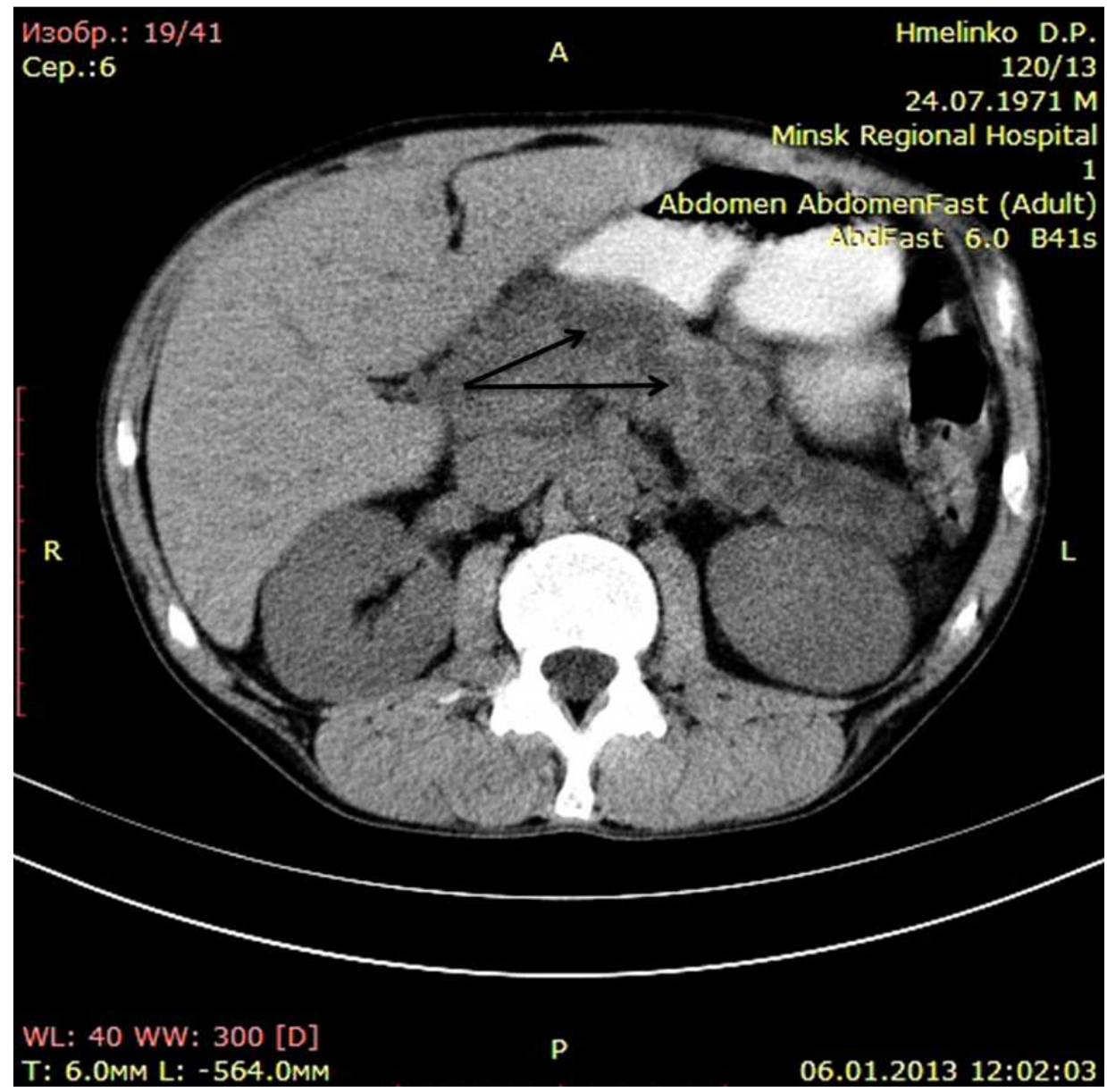

Figure 3. CT-scan before surgery: dilated tributary ducts in the body of pancreas (arrows).

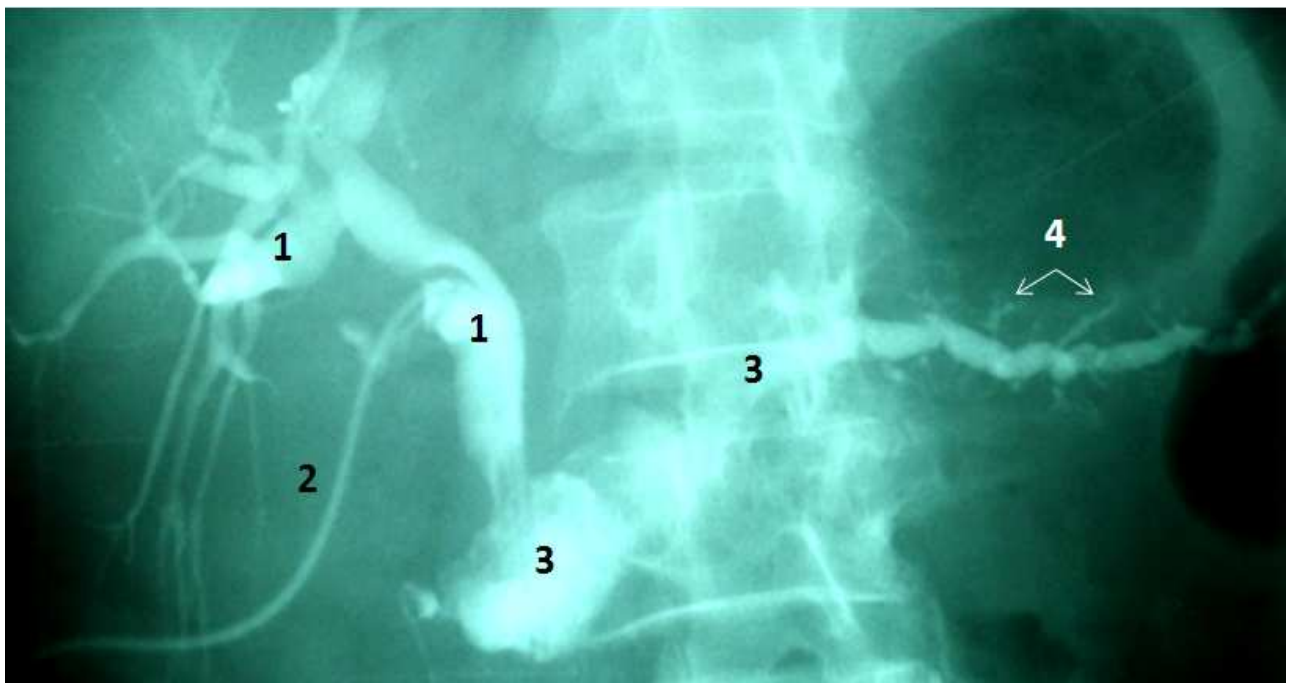

Figure 4. Fistulography of PJA lumen through the external drainage after performing the Berne's variant of Beger procedure and laser wirsungectomy: 1 - a biliary tree; 2 - drainage, inserted through the cystic duct and ChPS into the lumen of PJA; 3 - PJA lumen; 4 - the staining of the tributary ducts.

Classification of pancreatic duct stones. To characterize the concrements in the pancreatic ductal system, the term «wirsungolithiasis» is conventional. Consequently, it might be more correct to use a more general term «pancreatic ductal lithiasis», the term «pancreatoliths» being referred to the concrements.

We propose the following classification of «pancreatic ductal lithiasis».

According to the localization of the stones in the pancreatic ducts:

a) magistral ducts (wirsungolithiasis, santorini lithiasis, processus uncinati duct lithiasis)

b) second or third order ducts («tributarylithiasis», previously referred as «calcinates») 
c) the entire pancreatic ductal system - total pancreatic ductal lithiasis (Figure 5)

d) residual pancreatoliths in PJA lumen.

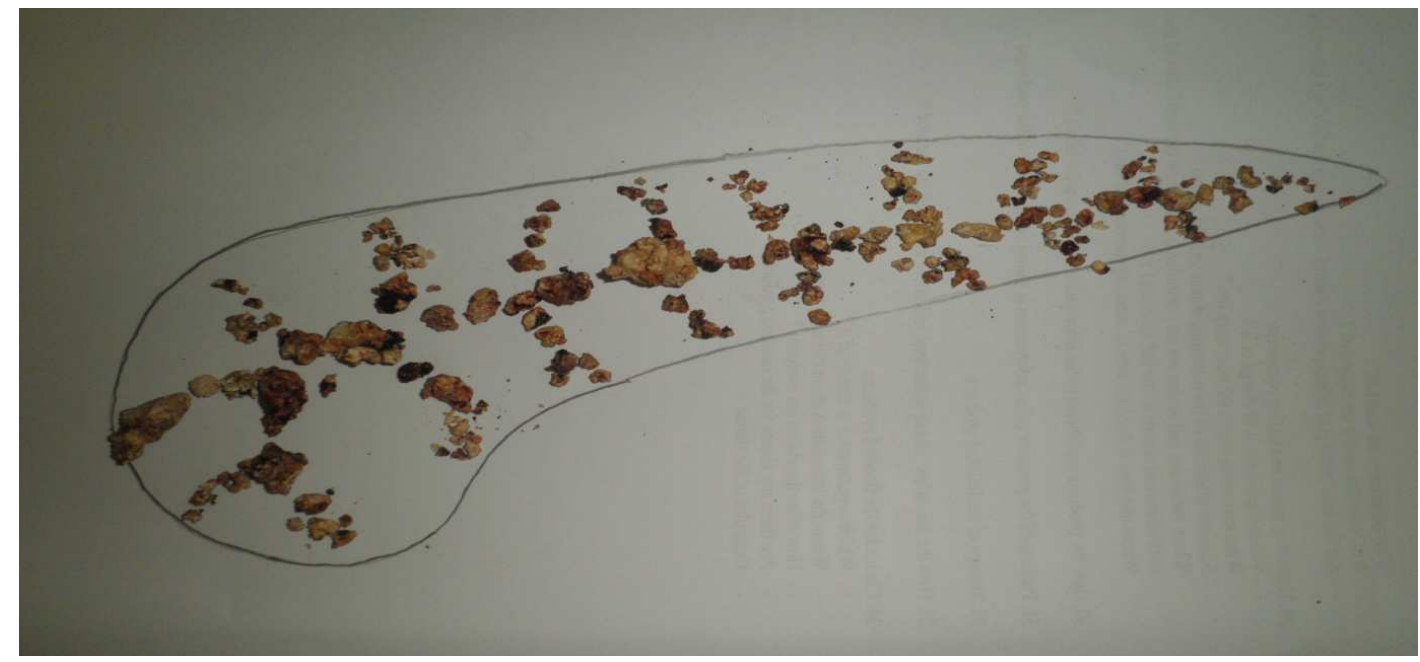

Figure 5. Reconstruction of own surgical macrospecimen: total pancreatic ductal lithiasis.

Types of ductal hypertension caused by pancreatoliths:

1. magistral

2. peripheral

3. combined

So, «calcinates» diagnosed by MRI-, CT- or US-scans must be referred as «tributaryliths» and, in the presence of intractable abdominal pain, should be considered as an indication for elective CP surgery. The effect of intrasurgical laser «cavitation» of the concrements during laser resection of the pancreas allows the residual tributaryliths falling out into PJA lumen. After this, the outflow of pancreatic juice into the lumen of PJA is restored that is confirmed by DBE examination after the surgery. In our series, $17,7 \%$ of patients presented with PDH. During the follow up (6 to 36 months) after the surgical treatment, $93,4 \%$ of patients remained pain free.

\section{Discussion}

There are two known causes of intractable chronic abdominal pain in CP: perineural inflammation and masses in the pancreatic head $[1,2,3]$ and magistral pancreatic hypertension due to strictures of Wirsung duct and/or wirsungoliths $[4,5,7,8]$. For removing the inflammatory masses in the pancreatic head, the Beger procedure and its Berne's modification are proposed showing good long-term results $[18,19]$. In the presence of two causes of chronic abdominalgia (inflammatory masses in the pancreatic head and magistral pancreatic hypertension), a modified Frey procedure is usually performed [17].

Our experience in repeated surgery of $\mathrm{CP}$ and the first data of DBE examinations of PJA lumen allow asserting that a currently used resection of the pancreatic head and Frey procedure can reduce only magistral duct hypertension. The concrements in the tributary ducts, earlier referred as «calcinates» in the parenchyma of the pancreas, are firmly fixed in the ducts due to their corral-like shape, blocking a free outflow of pancreatic juice into the lumen of PJA, thus resulting in «peripheral ductal hypertension» [25]. We introduced this term instead of «peripheral parenchymal pressure» or «glandular pancreatic compartment syndrome» $[7,8,9]$. The proposed classification of pancreatoductolithiasis can explain the causes of severe abdominal pain and its recurrence after traditional surgical procedures.

PDH causes an intractable abdominal pain after traditional drainage procedures for $\mathrm{CP}$. Therefore, repeated surgery of $\mathrm{CP}$ complicated by magistral and peripheral ductal hypertension should include the resection of the pancreatic head using Berne's modification of Beger procedure and a V-shaped resection of the pancreatic body and tail by Izbicki followed by PJ. This approach allows eliminating the both causes of ductal hypertension and relieving the patient from chronic abdominalgia.

We propose a pathogenetically based new primary surgical treatment for CP. When pancreatoliths or inflammatory masses located in the head and tributaryliths in the body of the pancreas are confirmed by MRI-, CT- or US-scans, two procedures should be performed - the resection of the pancreatic head by the Berne's procedure and the laser longitudinal pancreatowirsungotomy with laser cylindric wirsungectomy in the body of the pancreas [26].

\section{Conclusions}

1. DBE has great opportunities for diagnosing the complications after PJ.

2. Laser technologies significantly enhance the therapeutic effect of DBE, including the laser lithotripsy of residual pancreatoliths (Figure 6) and the vaporization of PJA and ChPS strictures.

3. Laser longitudinal pancreatowirsungotomy with laser cylindric wirsungectomy allows reducing the PDH in the body of the pancreas without further bleeding. As compared with Izbicki resection, this technique 
preserves more pancreatic tissue and prevents exo- and endocrine insufficiency.

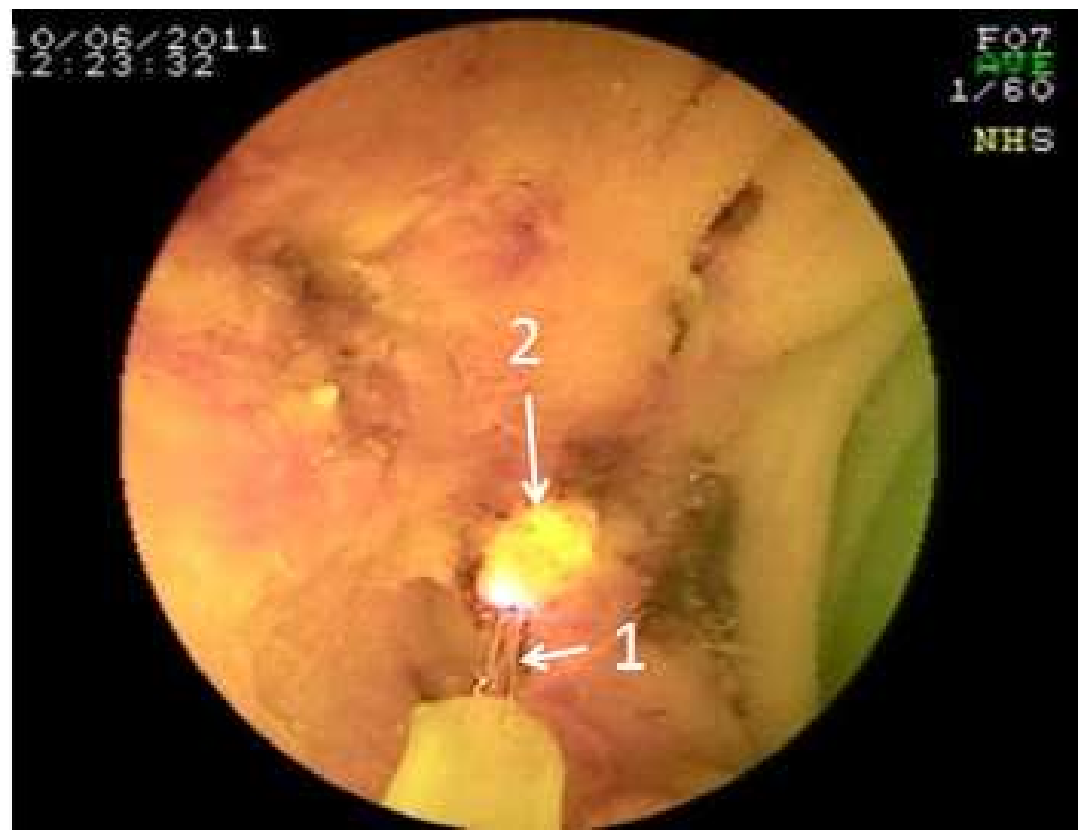

Figure 6. DBE laser lithotripsy (arrow 1) of the residual pancreatolith (arrow 2) in the lumen of PJA.

\section{References}

[1] Di Sebastiano P, Di Mola FF, Bockman DE, Friess H, Buchler MW. Chronic pancreatitis: the perspective of pain generation by neuroimmune interaction. Gut 2003; 52: 907-911.

[2] Bockman DE, Buchler M, Malfertheiner P, Beger HG. Analysis of nerves in chronic pancreatitis. Gastroenterology 1988; 94: 1459-1469.

[3] Buchler M, Weihe E, Friess H, Malfertheiner P, Bockman E, Muller S, Nohr D, Beger HG. Changes in peptidergic innervation in chronic pancreatitis. Pancreas 1992; 7 (2): 183-192.

[4] Bradley EL. Pancreatic duct pressure in chronic pancreatitis. Am J Surg 1982; 144: 313-316.

[5] Markowitz JS, Rattner DW, Warshaw AL. Failure of symptomatic relief after pancreatojejunal decompression for chronic pancreatitis. Arch Surg 1994; 129: 374- 380.

[6] Izbicki JR, Bloechle C, Broering DC, Kuechler T, Broelsch CE. Longitudinal V- shaped excision of the ventral pancreas for small duct disease in severe chronic pancreatitis: prospective evaluation of a new surgical procedure. Ann Surg 1998; 227: 213-219.

[7] Ebbehoj N, Svendsen LB, Madsen P. Pancreatic tissue pressure in chronic obstructive pancreatitis. Scand J Gastroenterol 1984; 19: $1066-1068$.

[8] Limrner JC, Knoefel WT, Bloechle C, Izbicki JR. Correlation between intraductal and intraparenchimatous pressure and pain in chronic pancreatitis [abstract], Int J Pancreatol 1996; 19: 237.

[9] Karanjia ND, Widdison AL, Leung FW. Compartment syndrome in experimental chronic obstructive pancreatitis: effect of decompressing the main pancreatic duct. Br J Surg 1994; 81: 259-264.
[10] Rios GA, Adams DB. Does intraoperative electrohydraulic lithotripsy improve outcome in the surgical management of chronic pancreatitis? Am Surg 2001; 67: 533- 537.

[11] 11.Sanerbruch T, Holl J, Sackman M, Paumgartner G. Extracorporal lithotripsy of pancreatic stones in patients with chronic pancreatitis and pain: a prospective follow- up study. Gut 1992; 33, 969-972.

[12] Ramesh H. Proposal of a new grading system for chronic pancreatitis: the ABC system. Clin Gastroenterol 2002; 35: $67-70$.

[13] Buchler M, Martignoni M, Friess H, Malfertheiner P. A proposal for a new clinical classification of chronic pancreatitis. Gastroenterol 2009; 9: 93-100.

[14] Schneider A, Lohr S, Singer M. The M-ANNHEIM classification of chronic pancreatitis: introduction of a unifying classification system based on a review of previous classifications of the disease. Gastroenterol 2007; 42: 1-19.

[15] Partington RF, Rochelle REL. Modified Puestow procedure for retrograde drainage of the pancreatic duct. Ann Surg 1960; 152: 1037-1042.

[16] Frey CF, Smith GJ. Description and rationale of a new operation for chronic pancreatitis. Pancreas 1987; 2: 701-707.

[17] Frey CF, Kathrin L, Mayer M. Comparison of local resection of the head of the pancreas combined with longitudinal pancreaticojejunostomy (Frey procedure) and duodenum-preserving resection of the pancreas head (Beger procedure). World J Surg 2003; 27: 1217-1230.

[18] Beger HG, Schlosser W, Friess HM, Buchler MW. Duodenum-preserving head resection in chronic pancreatitis changes the natural course of the disease: a single- center 26-year experience. Ann Surg., 1999; 230: 512-519.

[19] Gloor B, Friess H, Uhl W, Buchler M. A modified technique of the Beger and Frey procedure in patient with chronic pancreatitis. Digest Surg 2001; 18: 21-25. 
[20] Varabei A, Shuleika A, Arlouski Y, Vizhinis Y, Lagodich N. Tributaryliths as a reason of peripheral pancreatic hypertension in chronic pancreatitis. Proceeding of the $45^{\text {th }}$ Meeting of the EPC. Zurich. 26-29 June 2013. Pancreatol 13 (39): 556.

[21] Yamamoto H, Sugano K. A new method of enteroscopy - the double balloon method. Can J Gastroenterol 2003; 17: 4, 273-274.

[22] Koornstra JJ, Fry L, Monkemuller K. ERCP with balloon-assisted enteroscopy technique: A systematic review. DigDis 2008; 26: 324-329.

[23] Parlak E, Cicek B, Disibeyaz S. Endoscopic retrograde cholangiography by double balloon endoscopy in patients with Roux-en-Y hepaticojejunostomy. Surg Endosc 2009; 6: 45-47.
[24] Chu YC, Yang CC, Yen YH, Chen CH, Yueh SK Double-balloon enteroscopy application in biliary tract disease - its therapeutic and diagnosis function. Gastrointest Endosc 2008; 68 (3): 585-591.

[25] Varabei A, Arlouski Y, Vizhinis Y, Shuleika A, Lagodich N, Derkacheva N. The use of double balloon enteroscopy for diagnosis and treatment of strictures of hepaticojejunal anastomoses after primary correction of bile duct injuries. Videosurgery Miniinv 2014; 9 (2): 219-225.

[26] Varabei A, Shuleika A, Arlouski Y, Vizhinis Y, Lagodich N, Lurie V. Chronic pancreatitis: basing of peripheral pancreatic hypertension and method of its surgical correction. Proceedings of the $44 *$ Annual Meeting of the EPC. Prague. 22-23 June 2012: 40 . 